\title{
CONSTRAINTS ON THE ORIGIN OF THE FIRST LIGHT FROM SN 2014J
}

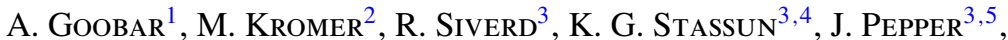 \\ R. Amanullah ${ }^{1}$, M. KaSliwal ${ }^{6}$, J. Sollerman ${ }^{2}$, AND F. TADDia ${ }^{2}$ \\ ${ }^{1}$ The Oskar Klein Centre, Department of Physics, Stockholm University, SE-106 91 Stockholm, Sweden; ariel@fysik.su.se \\ 2 The Oskar Klein Centre, Department of Astronomy, Stockholm University, SE-106 91 Stockholm, Sweden \\ ${ }^{3}$ Department of Physics and Astronomy, Vanderbilt University, VU Station B 1807, Nashville, TN 37235, USA \\ ${ }^{4}$ Physics Department, Fisk University, 1000 17th Avenue North, Nashville, TN 37208, USA \\ ${ }^{5}$ Department of Physics, Lehigh University, 413 Deming Lewis Lab, 16 Memorial Drive East, Bethlehem, PA 18015, USA \\ ${ }^{6}$ Observatories of the Carnegie Institution for Science, 813 Santa Barbara St, Pasadena, CA 91101, USA \\ Received 2014 October 8; accepted 2014 November 14; published 2015 January 20
}

\begin{abstract}
We study the very early light curve of supernova 2014J (SN 2014J) using the high-cadence broad-band imaging data obtained by the Kilodegree Extremely Little Telescope, which fortuitously observed M 82 around the time of the explosion, starting more than 2 months prior to detection, with up to 20 observations per night. These observations are complemented by observations in two narrow-band filters used in an $\mathrm{H} \alpha$ survey of nearby galaxies by the intermediate Palomar Transient Factory that also captured the first days of the brightening of the supernova. The evolution of the light curves is consistent with the expected signal from the cooling of shock heated material of large scale dimensions, $\gtrsim 1 R_{\odot}$. This could be due to heated material of the progenitor, a companion star or pre-existing circumstellar environment, e.g., in the form of an accretion disk. Structure seen in the light curves during the first days after explosion could also originate from radioactive material in the outer parts of an exploding white dwarf, as suggested from the early detection of gamma-rays. The model degeneracy translates into a systematic uncertainty of \pm 0.3 days on the estimate of the first light from SN 2014J.
\end{abstract}

Key word: supernovae: individual (SN 2014J)

\section{INTRODUCTION}

Type Ia supernovae (SNe Ia) rank among the sharpest tools to measure the expansion history of the universe. However, the ultimate limiting factor in their use for precision cosmology rests on our ability to control systematic uncertainties of astrophysical nature, most notably due to extinction and a possible brightness evolution over cosmic time (see Goobar \& Leibundgut 2011 for a recent review). Due to its remarkably close distance, SN 2014J in M $82(d \sim 3.5 \mathrm{Mpc})$ presents us with unique opportunities to study the environment and reddening of an otherwise normal SN Ia (Goobar et al. 2014; Amanullah et al. 2014; Kelly et al. 2014; Marion et al. 2014; Foley et al. 2014; Brown et al. 2014; Ashall et al. 2014), and even the impact of the intervening dust on the polarization signal (Patat et al. 2014; Kawabata et al. 2014). Moreover, its proximity allowed for the first time the direct detection of gamma-rays from the ${ }^{56} \mathrm{Ni}$ decay sequence (Churazov et al. 2014; Diehl et al. 2014) that power the optical display of SNe Ia. Contrary to expectations from most theoretical models, the detection of characteristic lines of the ${ }^{56} \mathrm{Ni}$ decay arising 20 days past explosion seems to indicate the presence of radioactive material close to the surface of the SN ejecta (Diehl et al. 2014).

Prior to this event, SN 2011fe in M $101(d \sim 6.4 \mathrm{Mpc})$ was the best studied SN Ia. In particular, Nugent et al. (2011) and Bloom et al. (2012) performed studies of the early light curve to conclude that the explosion originated from a compact object, $R \lesssim 0.02 R_{\odot}$, i.e., the size of a white dwarf (WD), as expected from models where SNe Ia result from a thermonuclear explosion in a C/O WD (Hoyle \& Fowler 1960). The validity of the tight limit has been questioned by Piro \& Nakar (2014), who suggested there could be a "dark phase" of up to a few days between the explosion and the rise of the radioactively powered light curve. Due to the lack of well-sampled deep observations prior to discovery, a possible early burst of light cannot be safely ruled out. Further discussions on the early rise of SNe Ia have been provided by Piro (2012), Piro \& Nakar (2013), and Dessart et al. (2014). From a spectroscopic analysis of SN 2011fe Mazzali et al. (2014) derived a dark phase of $\sim 1$ day, resulting in a limit of $R \lesssim 0.06 R_{\odot}$ for the progenitor size.

For SN 2014J, Zheng et al. (2014) reported January 14.75 UT ( \pm 0.21 day) as the best estimate of the explosion date, based on observations from the Katzman Automatic Imaging Telescope (KAIT) at Lick Observatory and amateur data from K. Itagaki. ${ }^{7}$ They also conclude that a fire-ball model (Riess et al. 1999), where the luminosity is assumed to scale with the area of the expanding photosphere at constant temperature and expansion velocity, $L \sim t^{2}$, does not provide a good description to the KAIT data of SN 2014J and used instead a broken power-law expression to derive the time of first light. Similarly, Goobar et al. (2014) showed that the early data of SN 2014J from the intermediate Palomar Transient Factory (iPTF) obtained with the 48 inch telescope (P48) in two narrow-band $\mathrm{H} \alpha$ filters deviate from the simple $t^{2}$ law.

In this work, we investigate the possibility that the deviations from a simple $t^{2}$ rise seen in the light curves of SN 2014J originate from an extra source of luminosity from either shockheating of the ejecta, interaction with circumstellar matter or a companion star; or from radioactive material in the outer parts of the exploding star. For this purpose, we use the early iPTF narrow-band data from Goobar et al. (2014) and the highcadence observations from the Kilodegree Extremely Little Telescope (KELT) from Siverd et al. (2015).

\footnotetext{
7 http://www.k-itagaki.jp/psn-m82.jpg
} 

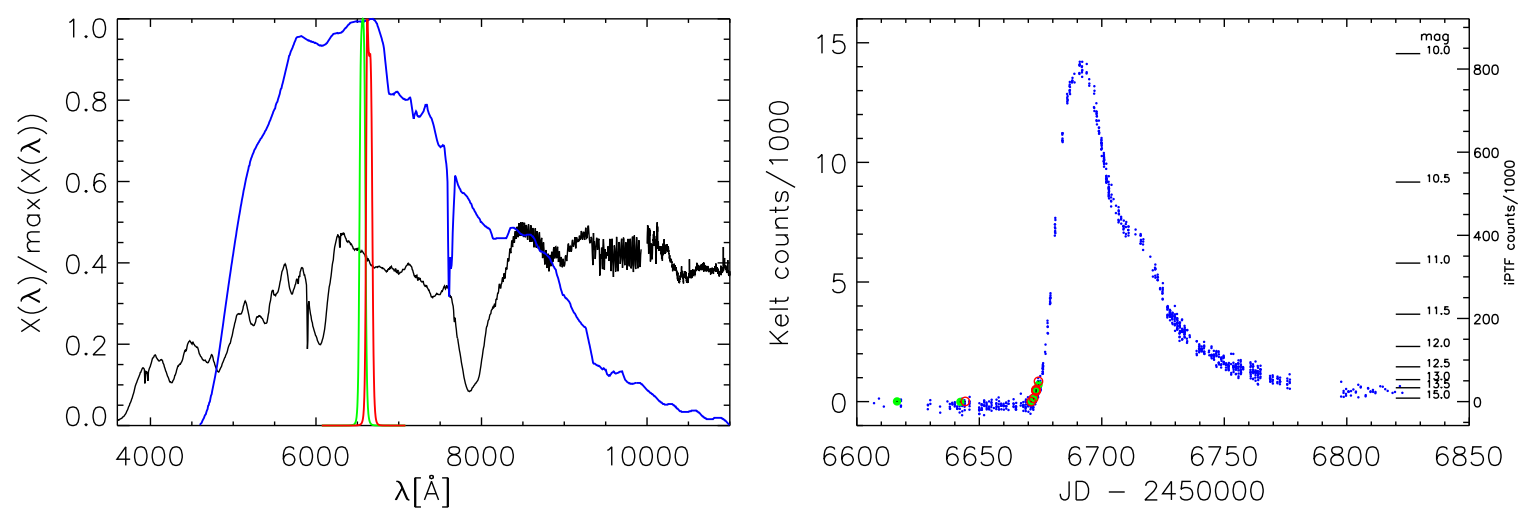

Figure 1. Left: system transmission functions $X(\lambda)$ for the KELT (broad-band; blue) and iPTF (narrow filters; green/red) used for the early observations of SN 2014J. Also shown in black is the earliest spectrum available, $F_{\lambda}$, from Cao et al. (2014), about seven days after the derived explosion date. Right: the KELT (blue) and iPTF (red, green) light curve points. KELT covers epochs from about two months prior to explosion to day 140 after peak. Also shown are the natural Vega magnitudes for the calibrated fluxes.

\section{OBSERVATIONS}

The effective filter pass-bands (i.e., including the telescope optics, CCD sensitivity, and atmospheric throughput) used for the early observations of SN 2014J from iPTF (Goobar et al. 2014) and KELT (Siverd et al. 2015) are shown in Figure 1, along with the SN 2014J classification spectrum (Cao et al. 2014) from January 22. The explosion occurred around the time of the full Moon, when iPTF was conducting a narrow-band survey of the Galactic plane and neighboring galaxies with the Palomar 48 inch telescope. Thus, the first set of iPTF imaging observations were conducted with $\mathrm{H} \alpha$ filters centered at $6564 \AA$ and $6638 \AA$, respectively. Also shown in Figure 1 is the full (currently) available KELT light curve, starting about two months prior to explosion and reaching to about day +140 after light curve maximum.

KELT is primarily an exoplanet transit survey (Pepper et al. 2007), using a small aperture telescope $(42 \mathrm{~mm})$ with a very wide field of view (FOV), $26^{\circ} \times 26^{\circ}$ and a broad transmission function, $X(\lambda)$, cutting around $4600 \AA$ on the blue side and reaching into the near-IR, $\lambda \sim 1 \mu \mathrm{m}$. The effective wavelength of the KELT pass-band (filter plus CCD response) is $\lambda_{\text {eff }} \approx$ $691 \mathrm{~nm}$. KELT has a very coarse pixel scale, $\sim 23^{\prime \prime}$, which makes accurate photometry challenging (Siverd et al. 2015). The KELT-North telescope obtained a total of 1869 individual exposures of SN 2014J that meet the quality control cuts. These exposures span 249 nights starting on 2013 October 8 and ending on 2014 June 14.

Because the KELT-North telescope uses a German Equatorial mount, observations taken before and after the local median crossing involve a $180^{\circ}$ flip of the FOV, which we designate west and east exposures. In order to avoid systematics in the flux scale between the west and east exposures, we restrict our analysis below to the west exposures. Therefore the final KELT light curve includes 889 individual exposures spanning 2013 November 10 to 2014 June 14 (Julian Dates 2,456,607.03 to $2,456,822.69)$. East and west are defined according to the sky position of the target, not by the orientation of the mount. In other words, the east exposures are acquired earlier in the night. There is in general an offset (and a scaling) difference between the east and west photometry. Consequently, the east and west data are reduced independently and have a different flux scale. We found empirically that the west data have a better behaved point-spread function (PSF) and smaller sky contamination, and therefore have overall better precision. Here we have opted to work with just the west orientation because of the better precision but most importantly to avoid systematics that could not be removed if attempting to also include the east data.

On nights with good weather where at least one measurement was obtained, the number of measurements per night varies from 1 to 20 . Note that the KELT observing procedure employs a Moon avoidance strategy, which leads to increased cadence on fields that are more than $60^{\circ}$ away from the Moon. Fortuitously, this Moon avoidance led to increased cadence on the M 82 field around the time of the explosion of SN 2014J. Flux measurements from the individual KELT-North frames are extracted using the difference-imaging aperture photometry pipeline described in Siverd et al. (2015), which is a modified version of the standard KELT pipeline (see Siverd et al. 2012). Briefly, the standard KELT pipeline was modified to deal with the non-optimal placement of M 82 near a corner of the KELT FOV, leading to poor extraction with the standard pipeline. Instead, in the modified pipeline, the photometry extraction was manually forced to a custom cutout region centered on M 82 . Each photometric measurement is a differential measurement relative to a template image by means of image subtraction. The template image is the median of 130 images obtained during the month prior to the explosion.

Typically, KELT achieves a photometric precision of $1 \%$ or better on point sources brighter than $V \approx 10$. In the case of SN 2014J, the KELT light curve achieved a photometric precision of $1.5 \%$ on average at peak brightness, and $3 \%$ on average at half peak brightness.

Photometry of the iPTF images was carried out with the method used in Amanullah et al. (2008). PSF photometry of the fluxes of all epochs together with the background host-galaxy light was fitted simultaneously. The degeneracy between the host and the SN was broken by fixing the SN flux to zero for all epochs obtained in 2013. Light curves were also built of 240 stars in the fields, which were used for calibration as well as for deriving systematic uncertainties between the epochs. The former was carried out by matching the field star photometry to SDSS DR $10 r^{\prime}$ and $i^{\prime}$ magnitudes. The uncertainty for individual epochs was found to be 0.03 mag and the uncertainty of the calibration is good to $0.07 \mathrm{mag}$. The photometry for the early rise used in this analysis is summarized in Table 1. 
Table 1

Data Used in This Analysis

\begin{tabular}{|c|c|c|}
\hline & $H_{\alpha}^{656}$ & Zero-point $=24.77$ \\
\hline UT 2014 Jan & Flux & Mag \\
\hline 14.18 & $-839.03 \pm 952.38$ & \\
\hline 15.18 & $6933.76 \pm 653.08$ & $15.17 \pm 0.08$ \\
\hline 16.17 & $23600.63 \pm 631.02$ & $13.84 \pm 0.02$ \\
\hline 16.25 & $25762.67 \pm 654.94$ & $13.74 \pm 0.02$ \\
\hline \multirow[t]{2}{*}{17.17} & $40287.14 \pm 566.79$ & $13.26 \pm 0.01$ \\
\hline & $H_{\alpha}^{663}$ & Zero-point $=24.83$ \\
\hline UT 2014 Jan & Flux & Mag \\
\hline 14.17 & $131.28 \pm 970.02$ & \\
\hline 15.18 & $7834.99 \pm 600.26$ & $15.09 \pm 0.07$ \\
\hline 16.17 & $25596.23 \pm 694.16$ & $13.81 \pm 0.02$ \\
\hline 16.17 & $28185.31 \pm 444.92$ & $13.70 \pm 0.01$ \\
\hline 16.22 & $28485.04 \pm 830.09$ & $13.69 \pm 0.03$ \\
\hline 16.22 & $26398.80 \pm 792.00$ & $13.78 \pm 0.03$ \\
\hline \multirow[t]{2}{*}{17.16} & $48755.88 \pm 713.46$ & $13.11 \pm 0.01$ \\
\hline & KELT & Zero-point $=20.41$ \\
\hline UT 2014 Jan & Flux & Mag \\
\hline 11.42 & $-241.71 \pm 67.48$ & \\
\hline 11.52 & $-24.08 \pm 80.86$ & \\
\hline 12.41 & $-70.10 \pm 79.43$ & \\
\hline 12.51 & $-140.29 \pm 89.18$ & \\
\hline 13.40 & $-177.58 \pm 79.28$ & \\
\hline 13.52 & $-139.61 \pm 76.93$ & \\
\hline 14.40 & $-73.86 \pm 75.38$ & \\
\hline 14.51 & $-162.87 \pm 75.99$ & \\
\hline 15.40 & $156.33 \pm 79.95$ & $14.92 \pm 0.44$ \\
\hline 15.51 & $161.54 \pm 89.63$ & $14.89 \pm 0.48$ \\
\hline 16.40 & $406.09 \pm 82.23$ & $13.89 \pm 0.18$ \\
\hline 16.50 & $622.89 \pm 98.03$ & $13.42 \pm 0.14$ \\
\hline 17.40 & $725.65 \pm 68.95$ & $13.25 \pm 0.08$ \\
\hline 17.50 & $600.53 \pm 76.19$ & $13.46 \pm 0.11$ \\
\hline 18.39 & $1316.60 \pm 66.64$ & $12.61 \pm 0.04$ \\
\hline 18.51 & $1428.99 \pm 77.22$ & $12.52 \pm 0.05$ \\
\hline
\end{tabular}

Notes. For KELT, only binned data is tabulated. The full data set is presented in Siverd et al. (2015).

\section{EARLY LIGHT CURVES OF SNe Ia}

Observationally, the early light curve shapes for $\mathrm{SNe}$ Ia follow, to a good approximation, a power-law behavior. For SN 2011fe, hitherto the object with the best-covered earlytime light curve, Nugent et al. (2011) found $L \propto t^{\beta}$, with $\beta=2.01 \pm 0.01$. Studies of stacked light curves of a nearby SN Ia sample from the LOSS survey (Ganeshalingam et al. 2011), typically not quite as sensitive to the very early phases, $t \lesssim 1$ day, found $\beta=2.20_{-0.19}^{+0.27}$. Similarly, a light curve analysis of SDSS-II SNe at cosmological distances by Hayden et al. (2010) found $\beta=1.8_{-0.18}^{+0.23}$, compatible with a similar study of SNLS SNe Ia, $\beta=1.8 \pm 0.2$, (Conley et al. 2006). Recently, Firth et al. (2014) studied a sample of 18 SNe Ia from the PTF and the La Silla-QUEST variability survey and found a mean value of $\beta=2.44 \pm 0.13$.

Nugent et al. (2011) and Piro (2012) have shown that such a power-law behavior can in principle result from radioactive heating of the SN ejecta. However, the exact shape of the rise will depend on the particular gradients in velocity, density, and chemical composition within the ejecta (Piro 2012). In particular, estimating the SN explosion epoch by fitting a power law to the early-time light curve might be misleading
(Piro \& Nakar 2014). Explosion models that can explain the observed properties of normal SNe Ia like delayed detonations in Chandrasekhar-mass WDs (e.g., Kasen et al. 2009; Sim et al. 2013; Dessart et al. 2014) or violent WD-WD mergers (Pakmor et al. 2012), predict that most ${ }^{56} \mathrm{Ni}$ should be buried in the central part of the ejecta and not reach to the outermost layers. As a consequence, it will take some time for the radioactive energy to diffuse to the surface of the ejecta and a dark phase might occur between the onset of explosion and the time of first light (Piro \& Nakar 2013, 2014).

\subsection{Cooling of Shock-heated Ejecta}

While the SN shock wave propagates through the progenitor star, it deposits energy all over the ejecta. Due to efficient adiabatic cooling, this shock deposited energy becomes negligible (with respect to radioactive heating) at $\sim 1$ day after explosion for a WD progenitor. At very early times, however, radiative diffusion from shock-heated outer layers of the SN ejecta may contribute to the emergent luminosity. In the past, several groups have investigated the cooling of shock heated SN ejecta with analytical frameworks, e.g., Chevalier (1992), Piro et al. (2010), Kasen (2010), Nakar \& Sari (2010), and Rabinak \& Waxman (2011). Assuming spherically symmetric, radiationdominated, homologously expanding ejecta with constant opacity, the different approaches vary in their treatment of radiative diffusion and opacity and their assumption of the initial density and pressure profiles. However, recent work has shown that the different approaches agree within a factor of $\sim 2$ (Rabinak \& Waxman 2011).

Here, we follow the approach in Nugent et al. (2011) and Bloom et al. (2012), who use the model of Rabinak \& Waxman (2011) to calculate the early-time luminosity $\left(L^{\mathrm{E}}\right)$ and temperature $\left(T^{\mathrm{E}}\right)$ from shock-heated ejecta:

$$
\begin{aligned}
L^{\mathrm{E}}(t)= & 1.2 \times 10^{40} R_{10}\left(E_{51}\right)^{0.85}\left(M_{\mathrm{c}}\right)^{-0.69}\left(\kappa_{0.2}\right)^{-0.85} \\
& \times\left(f_{\mathrm{p}}\right)^{-0.16}\left(t_{\mathrm{d}}\right)^{-0.31} \mathrm{erg} \mathrm{s}^{-1} \\
T_{\mathrm{eff}}^{\mathrm{E}}(t)= & 4.1 \times 10^{3}\left(R_{10}\right)^{0.25}\left(E_{51}\right)^{0.016}\left(M_{\mathrm{c}}\right)^{0.03}\left(\kappa_{0.2}\right)^{0.27} \\
& \times\left(f_{\mathrm{p}}\right)^{-0.022}\left(t_{\mathrm{d}}\right)^{-0.47} \mathrm{~K},
\end{aligned}
$$

where $R_{10}$ is the progenitor radius in units of $10^{10} \mathrm{~cm}, E_{51}$ the explosion energy in units of $10^{51} \mathrm{erg}, M_{\mathrm{c}}$ the progenitor mass in units of the Chandrasekhar mass $\left(1.38 M_{\odot}\right), \kappa_{0.2}$ the opacity in units of $0.2 \mathrm{~cm}^{2} \mathrm{~g}^{-1}, f_{\mathrm{p}}$ a form factor that depends on the density profile of the progenitor star, and $t_{\mathrm{d}}$ the time since explosion in days. In the following, we adopt $E_{51}=1, M_{\mathrm{c}}=1, \kappa_{0.2}=1$ and $f_{\mathrm{p}}=0.05$.

\subsection{Cooling of a Shock-heated Companion Star}

Similar to the situation discussed in the previous section, the SN shock wave will heat the surface layers of a surviving companion star, if present. This shock-deposited energy may then contribute to the early-time luminosity of the SN. This scenario was investigated by Kasen (2010). From a multidimensional radiative transfer simulation, Kasen showed that the emerging flux is strongly angle-dependent. For favorable viewing angles, i.e., those lying within the hole that is carved out of the SN ejecta by interaction between the ejecta and the companion star, shock heating of the companion star leads to a significant flux excess at early times compared to pure radioactive heating. Hydrodynamical simulations of interactions between the ejecta and the companion star show that the half 
opening angle of such ejecta holes is $\sim 30^{\circ}-40^{\circ}$ (e.g., Marietta et al. 2000; Pakmor et al. 2008). In contrast, from the opposite direction the optically thick ejecta are obscuring the companion and no effect of the shock heating is present, as shown in Figure 2 of Kasen (2010).

Kasen (2010) provides an analytic fit formula for the evolution of the early-time bolometric luminosity and temperature, $L^{\mathrm{C}}$ and $T^{\mathrm{C}}$ :

$$
\begin{aligned}
L^{\mathrm{C}}(t) & =10^{43} a_{13}\left(M_{\mathrm{c}}\right)^{1 / 4}\left(v_{9}\right)^{7 / 4}\left(\kappa_{\mathrm{e}}\right)^{-3 / 4}\left(t_{\mathrm{d}}\right)^{-1 / 2} \mathrm{erg} \mathrm{s}^{-1} \\
T_{\mathrm{eff}}^{\mathrm{C}}(t) & =2.5 \times 10^{4}\left(a_{13}\right)^{1 / 4}\left(\kappa_{\mathrm{e}}\right)^{-35 / 36}\left(t_{\mathrm{d}}\right)^{-37 / 72} \mathrm{~K} .
\end{aligned}
$$

Here, $a_{13}$ is the separation distance between the progenitor and its companion in units of $10^{13} \mathrm{~cm}, M_{\mathrm{c}}$ the progenitor mass in units of the Chandrasekhar mass, $v_{9}$ the expansion velocity of the SN ejecta in units of $10^{9} \mathrm{~cm} \mathrm{~s}^{-1}, \kappa_{\mathrm{e}}$ the electron scattering opacity in units of $0.2 \mathrm{~cm}^{2} \mathrm{~g}^{-1}$ and $t_{\mathrm{d}}$ the time since explosion in days. In the following we adopt $M_{\mathrm{c}}=1, \kappa_{\mathrm{e}}=1$ and $v_{9}=1$. Assuming that the companion fills its Roche lobe, its radius can be constrained to be $\lesssim 0.5 a_{13}$.

\section{ANALYSIS OF THE EARLY LIGHT CURVES OF SN 2014J}

Zheng et al. (2014) pointed out that the early broad-band light curve of SN 2014J does not follow a unique power law. Instead, a broken power-law expression was used to fit the data for the purpose of deriving the explosion date. Apart from SN 2014J, today there is only one other SN known that shows such a behavior (SN 2013dy; Zheng et al. 2013). For the bulk of observed SNe Ia, the light curve rise is in good agreement with a single power law (Conley et al. 2006; Hayden et al. 2010; Ganeshalingam et al. 2011).

In what follows, we will try to reconcile the deviant behavior of SN 2014J as being a combination of (at least) two effects: radioactive heating from the inner ejecta and some extra component, e.g., from shock-heating of the ejecta or a companion; or surface radioactivity, described in Section 4.4.

\subsection{The Fitting Procedure}

Given the empirical evidence for a power-law behavior for the radioactive early light curve of a large body of SNe Ia, we assume that the flux through a system transmission function $X(\lambda)$ (see Figure 1) can be described by the sum of two components, including one of the terms in Equations (1) or (2) and a power law:

$$
\mathcal{F}_{X}(t)= \begin{cases}C_{X}\left(t-t_{0}\right)^{\beta}+\int \frac{\mathcal{L}^{\delta}\left(\lambda, t-t_{0}\right)}{4 d^{2}} X(\lambda) 10^{\left(-\frac{A_{\lambda}}{2.5}\right)} d \lambda & t \geqslant t_{0} \\ 0 & t<t_{0},\end{cases}
$$

where $\delta$ is an index specifying the source of luminosity (e.g., heating of ejecta or companion star) and $A_{\lambda}$ corresponds to the adopted extinction law, where for SN 2014J we base our extinction corrections on the parameters in Amanullah et al. (2014), and $\mathcal{L}^{\delta}(\lambda, t)$ is the wavelength-dependent luminosity:

$$
\mathcal{L}^{\delta}(\lambda, t)=L^{\delta}(t) \cdot \frac{B_{\lambda}\left(T_{\mathrm{eff}}^{\delta}(t)\right)}{\sigma_{\mathrm{SB}}\left[T_{\mathrm{eff}}^{\delta}(t)\right]^{4}},
$$

where we have assumed a blackbody spectral energy distribution and used the Stefan-Boltzmann law.

Next, we use the above expressions to fit the measured light curves for the first four days after the explosion, along with preexplosion data to derive the explosion time, $t_{0}$ and the physical parameters of the extra source of energy. For the case of shockheated ejecta, we fit the radius of the progenitor star. When investigating the interaction with the companion, the parameter of interest is the radius of the companion star, assuming Rochelobe filling.

It should be noted that the adopted phenomenological description in Equation (3) cannot be exact since, at any given time, the two contributions will not be completely independent. E.g., the same parts of the ejecta may contribute due to shock heating and radioactive heating. However, in the limit of $t \rightarrow 0$ the shock heating will dominate, while for $t \rightarrow \infty$ radioactive heating is the main source of energy for the optical light curves. Similarly, the fitted power-law component could absorb some of the contributions of the extra source of power.

We choose to fit the data in flux space (not magnitudes) to minimize potential bias effects. This is important since for $t<t_{0}$ positive as well as negative fluctuations should be treated equally. Furthermore, the (mainly) Poisson noise is simple to characterize in linear flux space.

\subsection{Verification of the Method Using Observations of SN 2011 fe}

We first verify our method by revisiting the results on SN $2011 \mathrm{fe}$ based on the $g$-band magnitudes reported by Nugent et al. (2011), also adopting their assumed distance to M 101 of $6.4 \mathrm{Mpc}$ and negligible extinction $A_{\lambda}$. For the epochs with nondetections, we set the flux to zero and assign the (symmetric) $1 \sigma$ error bar from the reported upper limits in Table 1 of Nugent et al. (2011). The fit results are shown in the two top panels of Figure 2, where one can see that our results are in good agreement with Nugent et al. (2011) and Bloom et al. (2012). In particular, we find that a combination of a power-law model $(\beta=2)$ with shock heating of a compact progenitor of radius $R=0.02 R_{\odot}$ provides an excellent fit, as shown in Figure 2(a). For the interaction with the companion, as specified by the model of Kasen (2010) described in Section 3.2, we find in Figure 2(b) that a companion star of size $R \lesssim 0.25 R_{\odot}$ provides a good fit to the observed $g$-band light curve, i.e., slightly less constraining than the limits found by Bloom et al. (2012), $R<0.1 R_{\odot}$. The small discrepancy is likely connected to the different analysis approaches, i.e., the use of flux space and two components in the fit.

\subsection{Fits of the Combined iPTF/KELT Data of SN 2014J}

For the analysis of the early imaging data of SN 2014J we use the same methodology as for SN 2011fe in Section 4.2. For the system transmissions of iPTF and KELT we use the functions $X(\lambda)$ shown in Figure 1. Furthermore, we adopt the distance to M 82 from Dalcanton et al. (2009), $d=3.5$ $\mathrm{Mpc}$, and the host galaxy reddening of SN 2014J derived by Amanullah et al. (2014), where we opted to use their parameter solution for the Galactic type extinction model of Fitzpatrick (1999), $R_{V}=1.4, E(B-V)_{\text {host }}=1.37 \mathrm{mag}$. Changing to the multiple scattering model of Goobar (2008), found to give a slightly better fit to the full multi-wavelength light curve data from UV to the near-IR in Amanullah et al. (2014) and Foley et al. (2014), does not have a major impact on the outcome of the fits. We also include Milky Way reddening, $R_{V}=3.1$, $E(B-V)_{M W}=0.06$ mag. We have assumed the dust causing the reddening is sufficiently far from the site of the that there is no significant change in the reddening properties over time. The results of the fits for $\beta \equiv 2$ are shown in the two bottom panels of Figure 2. 

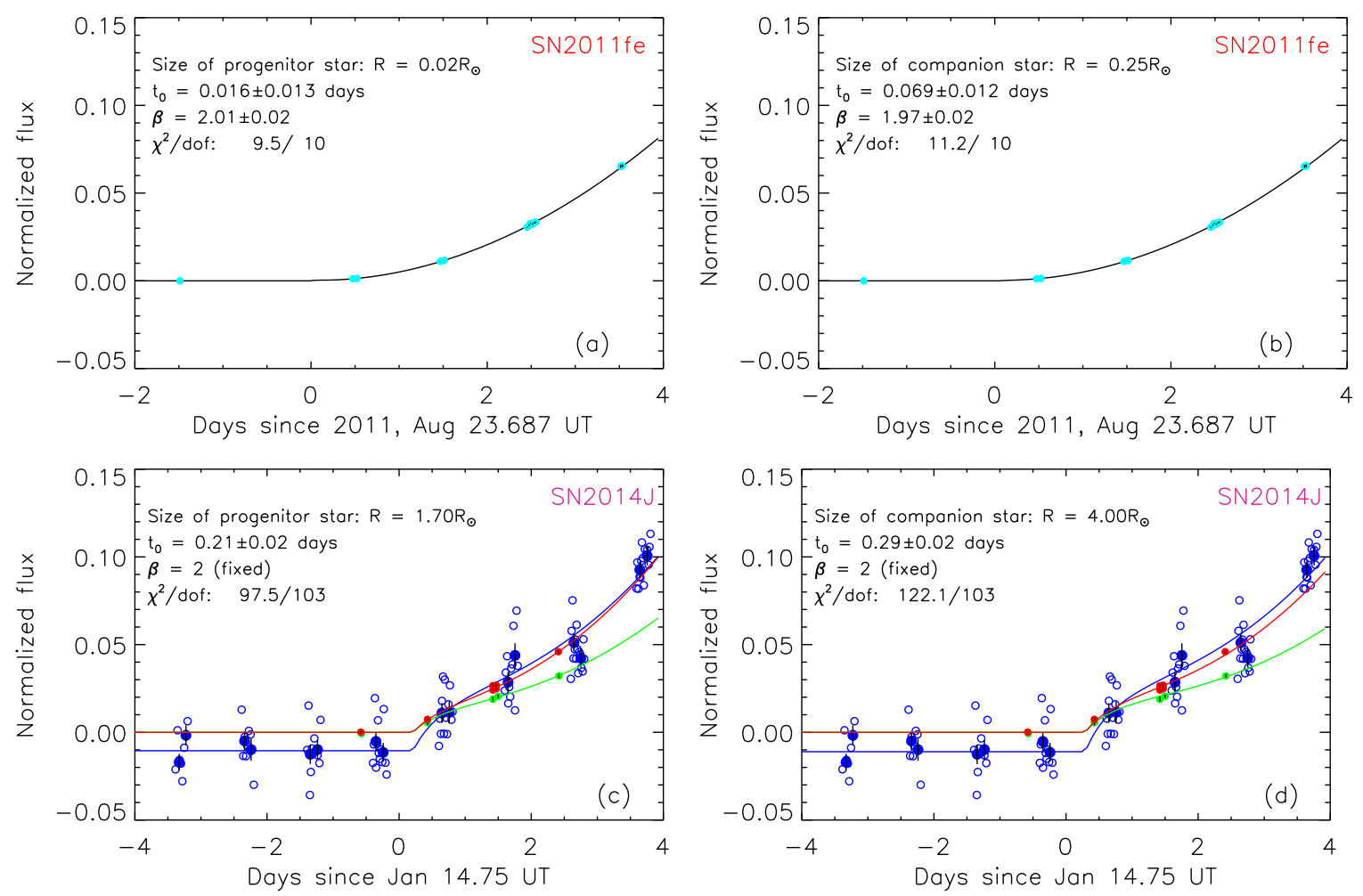

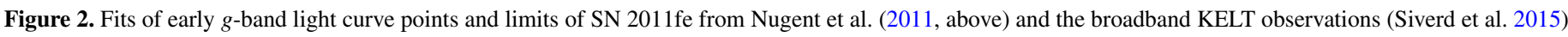

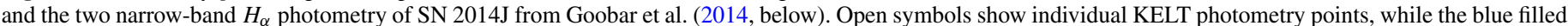

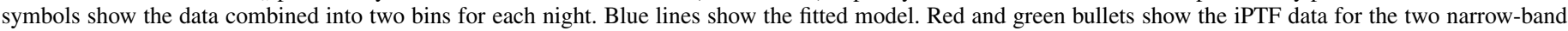

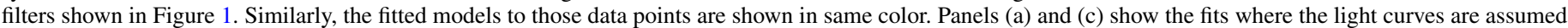

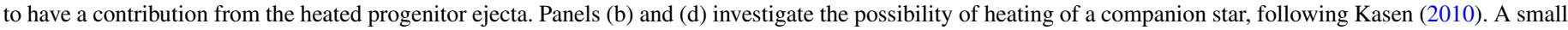
residual host galaxy contamination in the KELT photometry is fitted as a free (constant) parameter.

The light curves of the three filters are fitted simultaneously. The number of degrees of freedom and the $\chi^{2}$ for the best fit are also indicated in the figure. Clearly, the fits are statistically very sound and no systematic deviations are found for any of the data sets.

Since there is an arbitrary flux offset between the template image and the subsequent science images, this is accounted for in the light curve fitting through inclusion of a constant offset term. Note that the offset is in fact very small, comparable to the per-night binned photometric precision (i.e., smaller than the per-point photometric precision) and does not affect the results.

Fixing $\beta \equiv 2$, as observed in other SNe Ia, we find that for the scenario where the powering of the early rise of the light curve is a combination of a power-law and shock heating of the ejecta, the radius of the progenitor does not match that of a compact object for SN 2014J (at 90\% confidence level), unlike the case for SN $2011 \mathrm{fe}$. Instead, a radius in excess of the size of the Sun is required. For the assumptions made, we find a best fit for $R=1.7 R_{\odot}$. Levanon et al. (2014) argue that such large radii could be expected for WD mergers if an explosion does not occur on the dynamical timescale of the merger, as in the violent merger model (Pakmor et al. 2012), but on the longer viscous or thermal timescales of the merger remnant. In this case, angular momentum conservation leads to the formation of an accretion disk or extended envelope around the primary WD (Schwab et al. 2012) that extends to several solar radii. Whether such a configuration was present in the case of SN 2014J cannot be definitely assessed from our simple modeling. It is, however, interesting to note that Diehl et al. (2014) speculate about the formation of a $\mathrm{He}$ accretion belt to explain their detection of narrow gamma-ray lines from ${ }^{56} \mathrm{Ni}$ decay around maximum light. Detailed explosion models will be required to address this question.

When attributing the "bump" in the early light curve to heating of a companion star, we find that for the most optimal viewing angle (see Figure 2 in Kasen 2010) a companion of radius $4 R_{\odot}$ is required. For less favored viewing angles, much larger companion stars would be needed to fit the observations. We also find that, if this light curve modeling is assumed, the explosion occurred $7 \mathrm{hr}$ (0.29 days) later than using the fit based on a broken power-law light curve in Zheng et al. (2014). For the case of heated ejecta, our fits suggest that the explosion took place $5 \mathrm{hr}$ later than the best fit value of Zheng et al. (2014).

We note that the adopted formalism in Equation (3) implies a negligible dark phase, since the power-law component as well as the extra heating start at the same time, $t_{0}$. Although we could (in principle) add an extra free parameter in the fitting procedure to account for a time offset between the two sources of light, the excellent goodness of fit in our results does not motivate adding more degrees of freedom. We have also investigated the effect of combining our data set with the limits from Zheng et al. (2014) and find that this conclusion remains unchanged.

We note that it is still possible to get a formally acceptable fit for a compact progenitor and/or a small companion but only by invoking a very slow time dependence, $\beta=1.2-1.3$, as shown in Figure 3. According to Piro \& Nakar (2013), such a shallow rise is possible if direct heating from ${ }^{56} \mathrm{Ni}$ is dominating the luminosity indicating radioactive material right at the surface of the $\mathrm{SN}$ ejecta. This is also required to explain the detection of gamma-rays from ${ }^{56} \mathrm{Ni}$ decay as reported by Diehl et al. (2014). 

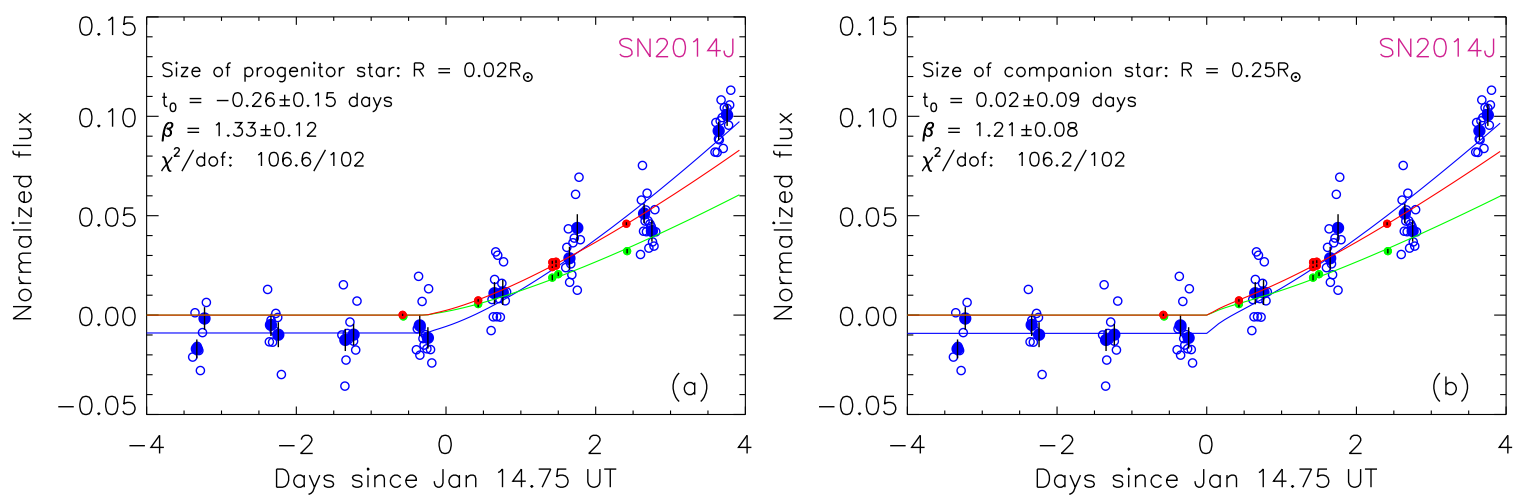

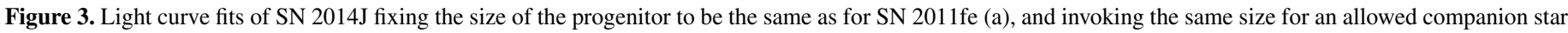

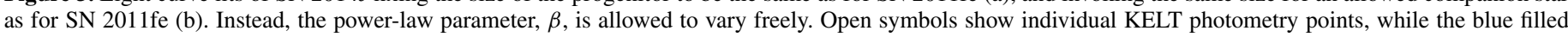

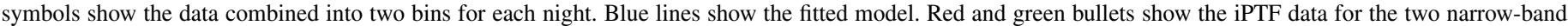

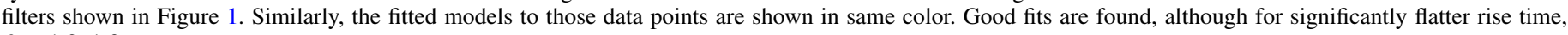
$\beta \sim 1.2-1.3$.

The explosion time is slightly perturbed, in the most extreme case by $6 \mathrm{hr}$ (earlier) than in Zheng et al. (2014).

\subsection{Surface Radioactivity}

In the following, we explore the possibility that radioactive material was deposited close to the surface of the SN ejecta in more detail. Surface radioactivity is actually predicted by various explosion models, e.g., turbulent deflagrations that lead to completely mixed explosion ejecta (e.g., Gamezo et al. 2003; Fink et al. 2014) or double detonations in sub-Chandrasekharmass WDs where an initial detonation in an accreted helium layer can lead to radioactive isotopes close to the surface of the ejecta (e.g., Kromer et al. 2010; Woosley \& Kasen 2011). How this material affects the early light curves in detail depends quite sensitively on its distribution and which isotopes are produced.

Piro \& Nakar (2013) presented a simplified analytical model to derive constraints on the ${ }^{56} \mathrm{Ni}$ distribution from the early bolometric light curve. Assuming that the radioactively powered early light curves scale with the amount of ${ }^{56} \mathrm{Ni}\left(M_{56}\right)$ that has been exposed at time $t$, the luminosity due to direct heating by ${ }^{56} \mathrm{Ni}$ is $L^{\mathrm{rad}}(t) \approx M_{56}(t) \cdot \epsilon(t)$, with:

$$
\epsilon(t)=\epsilon_{\mathrm{Ni}} e^{-t / t_{\mathrm{Ni}}}+\epsilon_{\mathrm{Co}}\left(e^{-t / t_{\mathrm{Co}}}-e^{-t / t_{\mathrm{Ni}}}\right),
$$

where $\epsilon_{\mathrm{Ni}, \mathrm{Co}}$ and $t_{\mathrm{Ni}, \mathrm{Co}}$ correspond to the specific heating and decay time of ${ }^{56} \mathrm{Ni}$ and ${ }^{56} \mathrm{Co}$, respectively.

For a steep gradient of radioactive material toward the inner parts of the exploding star, the deposition of gamma-rays could be dominated by deeper layers, with a fraction of photons escaping faster than the average diffusion time. Piro \& Nakar (2013) call this a "diffusive tail" (schematically shown in their Figure 2) and derive the expected contribution to the bolometric light curve:

$L^{\text {tail }}\left(t_{0} \leqslant t<t^{\prime}\right)=L^{\mathrm{rad}}\left(t^{\prime}\right) \frac{\epsilon\left(t-t_{0}\right)}{\epsilon\left(t^{\prime}-t_{0}\right)} \frac{\operatorname{erfc}\left(\left(t^{\prime}-t_{0}\right) / \sqrt{2}\left(t-t_{0}\right)\right)}{\operatorname{erfc}(1 / \sqrt{2})}$,

where "erfc" is the complementary error function. Next, we analyze the early light curve data of SN 2014J through fits of the theoretical models described above making the crude assumption that the narrow and broadband iPTF and KELT fluxes are good proxies for the bolometric luminosity, i.e., $L_{\text {bol }}(t) \propto L_{X}(t)$, where $X$ correspond to the filter data available.

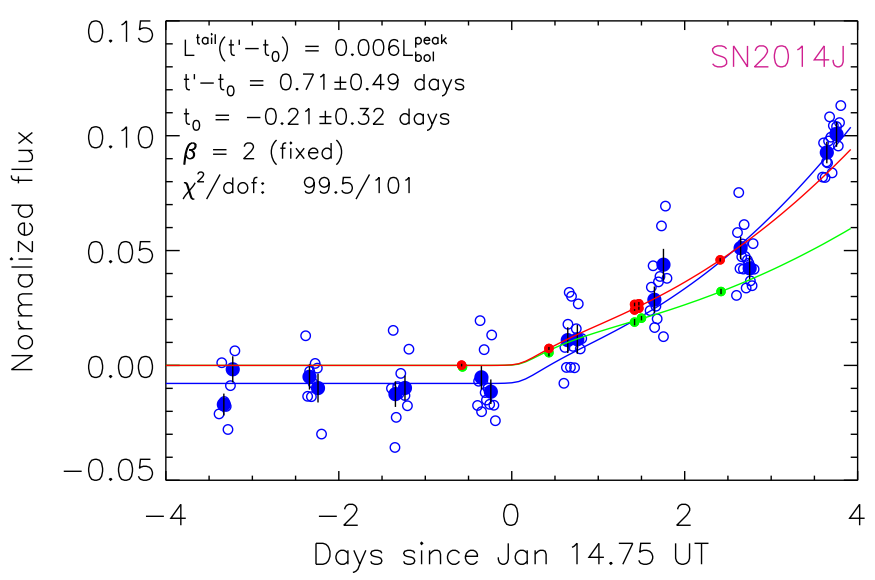

Figure 4. Light curve fits of SN 2014J assuming early rise has contributions from surface radioactivity Piro \& Nakar (2013), as described in Section 4.4. Open symbols show individual KELT photometry points, while the blue filled symbols show the data combined into two bins for each night. Blue lines show the fitted model. Red and green bullets show the iPTF data for the two narrowband filters shown in Figure 1. Similarly, the fitted models to those data points are shown in same color.

Following a similar approach as in Sections 3.1 and 3.2, we consider the observed fluxes to be the sum of two components:

$$
\mathcal{F}_{X}(t)=C_{X}^{1}\left(t-t_{0}\right)^{\beta}+C_{X}^{2} L^{\text {tail }}\left(t, t_{0}, t^{\prime}\right) .
$$

Here we should clarify that this treatment differs from Piro \& Nakar (2013), since their model aims at explaining the full light curve evolution, without the additional power-law term. Our treatment is empirically motivated by the good $\chi^{2}$ of the fit, as shown in Figure 4, which can be directly compared to the fits involving shock-heated material.

From the fitted luminosity, $L^{\text {tail }}\left(t^{\prime}-t_{0}\right)=6 \times 10^{-3} L_{\text {bol }}^{\text {peak }}$, and the assumption that the bolometric peak brightness is as for SN 2011fe, $L_{\mathrm{bol}}^{\text {peak }}=1.2 \times 10^{43} \mathrm{erg} \mathrm{s}^{-1}$ (Pereira et al. $2013)$, we can estimate the amount of "shallow" $\left(\sim 0.1 M_{\odot}\right.$ below surface) ${ }^{56} \mathrm{Ni}$ needed to power the light curve about 1 day after explosion, $M_{56} \approx 1.1 \times 10^{-3} M_{\odot}$, corresponding to a mixing mass fraction $X_{56} \approx 1.4 \times 10^{-2}$ when inserted in Equation 8 of Piro \& Nakar (2013). We note that this falls short to match the amount of surface ${ }^{56} \mathrm{Ni}$ invoked by Diehl et al. (2014) in their modeling of the detection of gamma-rays from SN 2014J. Given the possible degeneracy in our treatment, where the powerlaw component may absorb some of the contribution from 
radioactive surface material, our estimate may be regarded as a lower limit.

\section{CONCLUSIONS}

We have analyzed the early phase of the optical light curve following the explosion of SN 2014J in M 82. The high-cadence KELT data, with up to 20 visits per night, starting 2 months prior to the first detected light was used along with two sets of narrow-band data from iPTF. The observations are suitable for studies of the first few days following the explosion. This data set is unique among published light curves and can be compared to the previous nearest "normal" SN Ia in modern time, SN 2011fe.

Unlike the case for SN 2011fe, a $t^{2}$-model together with cooling from a compact progenitor does not provide the best match to the observations. Instead, we find that the required size scale of the heated material, either in the ejecta or a companion, is about a solar radius or larger. As the possibility of having a single degenerate model with a large companion has been ruled out by, e.g., searches in pre-explosion Hubble Space Telescope (HST) images (Goobar et al. 2014; Kelly et al. 2014); nondetections at X-ray (Margutti et al. 2014) and radio wavelengths (Pérez-Torres et al. 2014), other explanations to our findings are needed. Following Levanon et al. (2014), WD mergers could be one possibility. If ignited on a viscous timescale rather than a dynamical timescale, an extended circumstellar material will form around the primary WD. Shock heating of this material could be consistent with the observed early light curve of SN 2014J. Another possibility indicated by the shallow light curve rise could be that surface radioactivity contributes to the early light curve evolution. This was also invoked to explain the early gamma-ray signal from ${ }^{56} \mathrm{Ni}$ decay observed in SN 2014J by Diehl et al. (2014), albeit in larger quantities than was derived from our analysis of the optical light curve rise. Based on the available data, although with exquisite time sampling early on, the degeneracies between the various explanations cannot be resolved, due to insufficient signal-to-noise and color information. The model degeneracy translates into a systematic uncertainty of \pm 0.3 days on the estimate of the first light from SN 2014J.

We note that new well-sampled light curves are to be expected soon from the ongoing KEPLER extragalactic survey. ${ }^{8}$ As these will be carried on in an orchestrated fashion with surveys like iPTF and KAIT, we can expect future discoveries to have a richer spectroscopic and imaging coverage early on, e.g., in the UV from Swift and HST. Methods to distinguish between the various potential sources of luminosity in the early rise of Type Ia light curves, including temperatures and line velocities have been proposed by Piro \& Nakar (2014). We also note that similar attempts have been made to study the rise times of $\mathrm{Ib} / \mathrm{c}$ SNe in Taddia et al. (2014). Thus, the prospects of progress in this area are good.
A.G. and R.A. acknowledge support from the Swedish Research Council and the Swedish Space Board.

\section{REFERENCES}

Amanullah, R., Goobar, A., Johansson, J., et al. 2014, ApJL, 788, L21 Amanullah, R., Stanishev, V., Goobar, A., et al. 2008, A\&A, 486, 375 Ashall, C., Mazzali, P., Bersier, D., et al. 2014, MNRAS, 445, 4424 Bloom, J. S., Kasen, D., Shen, K. J., et al. 2012, ApJL, 744, L17 Brown, P. J., Smitka, M. T., Wang, L., et al. 2014, arXiv:1408.2381 Cao, Y., Kasliwal, M. M., \& McKay, A. 2014, ATel, 5786, 1 Chevalier, R. A. 1992, ApJ, 394, 599

Churazov, E., Sunyaev, R., Isern, J., et al. 2014, Natur, 512, 406 Conley, A., Howell, D. A., Howes, A., et al. 2006, AJ, 132, 1707 Dalcanton, J. J., Williams, B. F., Seth, A. C., et al. 2009, ApJS, 183, 67 Dessart, L., Blondin, S., Hillier, D. J., \& Khokhlov, A. 2014, MNRAS, 441,532

Diehl, R., Siegert, T., Hillebrandt, W., et al. 2014, Sci, 345, 1162

Fink, M., Kromer, M., Seitenzahl, I. R., et al. 2014, MNRAS, 438, 1762

Firth, R. E., Sullivan, M., Gal-Yam, A., et al. 2014, arXiv:1411.1064 Fitzpatrick, E. L. 1999, PASP, 111, 63

Foley, R. J., Fox, O. D., McCully, C., et al. 2014, MNRAS, 443, 2887

Gamezo, V. N., Khokhlov, A. M., Oran, E. S., Chtchelkanova, A. Y., \& Rosenberg, R. O. 2003, Sci, 299, 77

Ganeshalingam, M., Li, W., \& Filippenko, A. V. 2011, MNRAS, 416, 2607

Goobar, A. 2008, ApJL, 686, L103

Goobar, A., Johansson, J., Amanullah, R., et al. 2014, ApJL, 784, L12

Goobar, A., \& Leibundgut, B. 2011, ARNPS, 61, 251

Hayden, B. T., Garnavich, P. M., Kessler, R., et al. 2010, ApJ, 712, 350

Hoyle, F., \& Fowler, W. A. 1960, ApJ, 132, 565

Kasen, D. 2010, ApJ, 708, 1025

Kasen, D., Röpke, F. K., \& Woosley, S. E. 2009, Natur, 460, 869

Kawabata, K. S., Akitaya, H., Yamanaka, M., et al. 2014, ApJL, 795, L4

Kelly, P. L., Fox, O. D., Filippenko, A. V., et al. 2014, ApJ, 790, 3

Kromer, M., Sim, S. A., Fink, M., et al. 2010, ApJ, 719, 1067

Levanon, N., Soker, N., \& García-Berro, E. 2014, arXiv:1408.1375

Margutti, R., Parrent, J., Kamble, A., et al. 2014, ApJ, 790, 52

Marietta, E., Burrows, A., \& Fryxell, B. 2000, ApJS, 128, 615

Marion, G. H., Sand, D. J., Hsiao, E. Y., et al. 2014, arXiv:1405.3970

Mazzali, P. A., Sullivan, M., Hachinger, S., et al. 2014, MNRAS, 439, 1959

Nakar, E., \& Sari, R. 2010, ApJ, 725, 904

Nugent, P. E., Sullivan, M., Cenko, S. B., et al. 2011, Natur, 480, 344

Pakmor, R., Kromer, M., Taubenberger, S., et al. 2012, ApJL, 747, L10

Pakmor, R., Röpke, F. K., Weiss, A., \& Hillebrandt, W. 2008, A\&A, 489, 943

Patat, F., Taubenberger, S., Cox, N. L. J., et al. 2014, arXiv:1407.0136

Pepper, J., Pogge, R. W., DePoy, D. L., et al. 2007, PASP, 119, 923

Pereira, R., Thomas, R. C., Aldering, G., et al. 2013, A\&A, 554, A27

Pérez-Torres, M. A., Lundqvist, P., Beswick, R. J., et al. 2014, ApJ, 792, 38

Piro, A. L. 2012, ApJ, 759, 83

Piro, A. L., Chang, P., \& Weinberg, N. N. 2010, ApJ, 708, 598

Piro, A. L., \& Nakar, E. 2013, ApJ, 769, 67

Piro, A. L., \& Nakar, E. 2014, ApJ, 784, 85

Rabinak, I., \& Waxman, E. 2011, ApJ, 728, 63

Riess, A. G., Filippenko, A. V., Li, W., et al. 1999, AJ, 118, 2675

Schwab, J., Shen, K. J., Quataert, E., Dan, M., \& Rosswog, S. 2012, MNRAS, 427, 190

Sim, S. A., Seitenzahl, I. R., Kromer, M., et al. 2013, MNRAS, 436, 333

Siverd, R. J., Beatty, T. G., Pepper, J., et al. 2012, ApJ, 761, 123

Siverd, R. J., Goobar, A., Stassun, K. G., \& Pepper, J. 2015, ApJ, 799, 105

Taddia, F., Sollerman, J., Leloudas, G., et al. 2014, arXiv:1408.4084

Woosley, S. E., \& Kasen, D. 2011, ApJ, 734, 38

Zheng, W., Shivvers, I., Filippenko, A. V., et al. 2014, ApJL, 783, L24

Zheng, W., Silverman, J. M., Filippenko, A. V., et al. 2013, ApJL, 778, L15

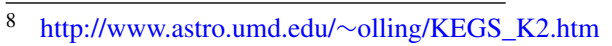

